\title{
Impact of Diamond Seeding on the Microstructural Properties and Thermal Stability of GaN-on-Diamond Wafers for High-Power Electronic Devices
}

\author{
Dong Liu, ${ }^{1,2}$ Daniel Francis, ${ }^{3}$ Firooz Faili, ${ }^{3}$ Callum Middleton, ${ }^{1}$ Julian Anaya, ${ }^{1}$ \\ James W. Pomeroy, ${ }^{1}$ Daniel J. Twitchen, ${ }^{3}$ and Martin Kuball ${ }^{1, a)}$ \\ ${ }^{1}$ Center for Device Thermography and Reliability, H.H. Wills Physics Laboratory, University of Bristol, Bristol \\ BS8 1TL, United Kingdom \\ ${ }^{2}$ Department of Materials, University of Oxford, Oxford OX1 3PH, United Kingdom \\ ${ }^{3}$ Element-Six Technologies, Santa Clara, CA 95054, USA
}

The impact of seeding of the diamond growth on the microstructural properties of GaN-on-diamond wafers was studied using in situ focused ion beam cross-sectioning and scanning electron microscopy imaging. Microstructural studies revealed that the seeding conditions are a critical parameter to obtain an optimal material, allowing the manufacture of GaN-on-diamond wafers with no microscopic defects and with structural stability under thermal annealing at $825^{\circ} \mathrm{C}$. The use of the right seeding conditions also results in homogeneous thermal properties across four inch GaN-on-diamond wafers, which is of critical importance for their use for ultra-high power microwave electronic devices.

Keywords: Compound semiconductors, diamond films, microstructure, thermal conductivity.

\footnotetext{
a) Authors to whom correspondence should be addressed: Martin.Kuball@bristol.ac.uk
} 
$\mathrm{AlGaN} / \mathrm{GaN}$-on-diamond microwave devices have demonstrated at least three times higher power density than devices grown on $\mathrm{SiC}$ substrates [1,2]. Since GaN-on-diamond substrates were first demonstrated [3], various groups have shown that diamond as substrate permits a more compact device design, enabling reduced gate finger spacing compared to devices on $\mathrm{SiC}$ substrates $[1,4,5]$. This allows for a dramatic shrinkage in monolithic microwave integrated circuits (MMIC) or power amplifier (PA) size and hence an increase in their efficiency through the removal of lossy combining networks. All this is due to the thermal conductivity of diamond, as high as $2000 \mathrm{~W} / \mathrm{mK}$ at $300 \mathrm{~K}$ compared to $450 \mathrm{~W} / \mathrm{mK}$ for SiC, which improves the waste heat extraction from the active device area [6]. The AlGaN/GaN device layers for AlGaN/GaN-on-diamond devices typically originate from a qualified epitaxy, as for example grown on Si substrates. Diamond growth or wafer-bonding is then used to replace the original substrate - which is first removed - with diamond $[1,7,8]$. To enable large-scale integration (> four inch wafers), the diamond is subsequently grown on the $\mathrm{N}$-face of the (Al)GaN epitaxial layers, using a thin dielectric layer to allow the seeding of the diamond onto the GaN [1]. This makes the GaN/diamond interface the critical region to be optimized in order to maximize the performance of devices. In recent years significant improvements in the thermal transport properties of the GaN/diamond interface have been achieved; for example the optimization of the GaN/diamond interfacial dielectric layer and the improved thermal properties of diamond near its nucleation site at the GaN/diamond interface [9-11]. As a consequence of these advances in understanding, it is now considered possible to achieve the best device characteristics by further optimizing the thermal transport at and/or near the GaN/diamond interface. This can be realized by adjusting the grain size of the diamond crystallites to be as large as possible near the nucleation region for maximized thermal conductivity [11] while the dielectric layer used to seed the diamond is kept as thin as possible to minimize its thermal resistance [9]. A straightforward strategy to adjust the grain size, targeted in this work, which has not yet been explored, is to modify the size of the diamond seeds used to grow the diamond underneath the GaN epitaxial layers. However, this strategy might impact the integrity of the GaN/diamond interface by promoting the formation of defects and voids which may impact the thermal and mechanical properties of the interface which is unknown. We emphasize that the mechanical properties are extremely crucial for the reliability of the material [12] since this interface is prone to be affected by the significant coefficient of thermal lattice expansion (CTE) mismatch between the diamond and the GaN. In this letter, the implication of different diamond seeding strategies, namely using larger and smaller seeding nanoparticles, on the ultimate microstructure, thermal properties of GaN-on-diamond wafers and its reliability are addressed. We demonstrate that GaN-on-diamond materials can be free from microscopic defects and have good homogenous thermal performance, however, this can only achieved if an appropriate diamond seeding approach is used.

For the GaN-on-diamond wafers studied, $\mathrm{AlGaN} / \mathrm{GaN}$ heterostructures grown on Si substrates by metal-organic chemical vapor deposition (MOCVD) were used as the starting material. A SiN dielectric layer was deposited on top of the structure to protect the device layer surface. The Si substrate and the AlGaN strain relief layer were then removed; a post grind and lap 
clean step was used to ensure that there is no residual AlGaN prior to the deposition of a $30 \mathrm{~nm}$ thin amorphous dielectric layer by low-pressure CVD for diamond seeding. As diamond growth is in a harsh environment with atomic hydrogen and high temperatures, the dielectric layer protects the GaN during this process. Diamond nanoparticles with two different average sizes, $30 \mathrm{~nm}$ and $100 \mathrm{~nm}$ diameter, were used for the seeding of the diamond growth, respectively. For the 30nm seeding, seeding density was better than $1 \times 10^{11} \mathrm{~cm}^{-2}$; for the $100 \mathrm{~nm}$ seeding, the density was about $1 \times 10^{10} \mathrm{~cm}^{-2}$. In both cases, a $100 \mu \mathrm{m}$ thick diamond layer was grown using microwave (MW) plasma CVD. Further details on the GaN-on-diamond structure and sample fabrication can be found in Ref. [11]. As-manufactured samples, and samples annealed at $825^{\circ} \mathrm{C}$ in nitrogen (temperature ramped up in 90 s, hold for 20 s, then cool down to room temperature over a period of 60s) were studied since this is an annealing condition typically used during the processing of devices for ohmic contact formation.

Characterization of the wafers was undertaken in a FEI Helio NanoLab 600i Dualbeam workstation. In particular, trenches were etched into the wafer by focused $\mathrm{Ga}^{+}$beam milling at a number of locations to create cross-sections of the GaN-ondiamond structure; a voltage of $30 \mathrm{kV}$ and current of $6.5 \mathrm{nA}$ was used, and this was followed by a final cleaning step with a much lower current, $48 \mathrm{pA}$. Cross-sections of the GaN-on-diamond structure were then reviewed and the microstructure characteristics evaluated by scanning electron microscope (SEM) imaging. All these cross-section images were undertaken at a stage tilt of $52^{\circ}$ to allow the in situ observation of the surface features as well as the cross-sections. In addition, the thermal properties of the GaN-on-diamond wafers were mapped by transient thermoreflectance and correlated to local microstructural features. A heating pulse from a $355 \mathrm{~nm}$ frequency-tripled $\mathrm{Nd}$ :YAG laser $(3.49 \mathrm{eV}$, i.e., above GaN bandgap) was absorbed into the GaN, with a pulse duration of $10 \mathrm{~ns}$ inducing a surface temperature rise. The resulting change in surface reflectance was monitored by a continuous wave (CW), $532 \mathrm{~nm}$ frequency-doubled Nd:YAG laser to track this temperature rise. From this the thermal boundary resistance ( $\mathrm{TBR}_{\mathrm{eff}}$ ) of the GaN/diamond interface could be determined. Heating and probe laser spots size were $60 \mu \mathrm{m}$ and $2 \mu \mathrm{m}$ respectively. Further details on this technique can be found in Ref. [13].

In general, for specimens grown with $100 \mathrm{~nm}$ diamond seeds, two types of defects were observed; denoted in the following as Type A and Type B. Type A, as shown in Fig. 1, appear as dark dots on the surface of the as-manufactured material under SEM imaging. These dots tend to follow straight lines and some are randomly distributed, Fig. 1a. Focused ion beam milling was used to cut trenches across these dark dots to reveal the cross-sectional features. It was found that these dark dots correspond to voids at the GaN/diamond interface, Fig. 1b. Some of these voids are of a size equivalent to the thickness of the full GaN layer (900nm in this case), i.e. penetrate to the surface (through-holes), while others are much smaller and remain concentrated at the $\mathrm{GaN} /$ diamond interface. After annealing at $825^{\circ} \mathrm{C}$, however, a larger number of these dark dots was found on the surface; cracks also formed, Fig. 1c, usually aligned with a line of dark dots, but they can deviate at defined angles. FIB cross-sectioning on these annealed samples indicates that with annealing (Fig. 1d) local debonding occurred; the interfacial voids have grown 
in size. In addition to an interfacial GaN/diamond debonding at the sites of the voids, other factors such as residual stresses in the GaN layer may have contributed to the propagation of cracks along cleavage planes of the GaN. Small voids also formed inside the diamond near the interface at these locations. Type B defects are nano-sized pinholes in the dielectric layer at the GaN/diamond interface in the as-manufactured material, Fig. 2a. In some areas, cavities along the interface form with 'teethlike' features between GaN and the diamond layer, Fig. 2b. These features are observed in both as-manufactured samples and after annealing.

As the through-holes (Type A defects) are mostly arranged in lines, this strongly suggests a mechanical abrasion process damaging the dielectric seed layer when the $100 \mathrm{~nm}$ diamond seeds are deposited. When the material structure is subsequently exposed to the diamond growth environment of hydrogen and high temperature, the GaN beneath the pinholes is etched, resulting in the formation of initial voids. During the continued diamond growth, these voids enlarge and evolve towards the top surface of the structure and can ultimately form through-holes that are visible from the surface of the material structure. As illustrated in Fig. 1d, annealing leads to the further expansion of the voids; a possible explanation for this is that the hydrogen stored at grain boundaries in the diamond near the GaN/diamond interface is released and etches the GaN at locations where voids are already present. In addition to the reduced structural and thermal stability of the material as a consequence of the defects, vertical leakage through the completed device structure was also observed - this is detrimental for the device performance [14].

For the specimens seeded with $30 \mathrm{~nm}$ size particles, shown in Fig. 3, no features were observable on the GaN surface, i.e. no through-holes or dark dots were present in the GaN layer after the diamond growth. In addition, voids were no longer present in the GaN near the interface to the diamond, Figs. 3a and 3b. Some undulations in the dielectric layer were still observable, however, not to the extent of creating any pinholes within the layer. This dielectric layer is considered essential to protect GaN from the harsh diamond growth environment, inhibiting the formation of voids and through-holes in the GaN. Furthermore, no microstructural changes were observed in the specimen subjected to annealing at $825^{\circ} \mathrm{C}$, neither the formation of voids, cracks or other microscopic features. Good stability of the GaN-on-diamond material was reported in Ref. [12] up to $950^{\circ} \mathrm{C}$. This demonstrates that changes in the seeding can produce GaN-on-diamond free of microscopic defects which is stable up to temperatures which are commonly used for $\mathrm{GaN}$ microwave device processing.

To assess the thermal homogeneity of the wafers and whether a non-invasive optical technique could be used to screen for voids in the material at the GaN/diamond interface, transient thermoreflectance measurements were performed. A TBR eff map obtained from the transient reflectance data is displayed in Fig. 4a for a wafer seeded with $30 \mathrm{~nm}$ size particles. Seeding with the smaller grains, i.e. by preventing the defect formation, resulted in a homogeneous $\mathrm{TBR}_{\text {eff }}$ of the GaN/diamond interface across the four inch wafer, with an average $\mathrm{TBR}_{\text {eff }}$ of $23 \pm 3 \mathrm{~m}^{2} \mathrm{~K} / \mathrm{GW}$. On the other hand, when the bigger grains are used for 
seeding the diamond, the defects such as voids at the GaN/diamond interface were apparent in clear non-ideal thermal reflectance transients as shown in Fig. 4b indicating inhomogeneous thermal properties. This demonstrates that this optical non-destructive measurement method can also be used for defect identification, i.e. for wafer screening after manufacture. $\mathrm{TBR}_{\text {eff }}$ determined for this wafer was in the range of $39-46 \mathrm{~m}^{2} \mathrm{~K} / \mathrm{GW}$, showing that the damage caused by the larger seeds led to an increased variation in the boundary quality. As these values are higher than that for the sample with smaller seeds, this implies that any benefit for the investigated samples afforded by the larger seed crystals appears mitigated by other factors affecting the GaN diamond interface, such as the defects discussed above.

In conclusion, the impact on the microstructure and thermal properties of GaN-on-diamond wafers when using different diamond seeding sizes ( $30 \mathrm{~nm}$ and $100 \mathrm{~nm}$ in diameter) to grow the diamond substrate has been investigated. It was found that the size of the diamond seeds plays an important role in the material microstructural and thermal properties and stability. Diamond seeds of the size of $30 \mathrm{~nm}$ diameter prevent the formation of microscopic defects and enable the manufacture of GaNon-diamond material free of obvious defects such as voids. In terms of the annealing robustness, the GaN-on-diamond wafer created with smaller diamond seeds is stable for annealing at temperatures of $825^{\circ} \mathrm{C}$. It showed homogenous thermal properties across the full wafer. In contrast, microscopic defects are present in GaN-on-diamond wafers created with larger diamond seeds; further annealing treatment promotes the enlargement of those interfacial defects. In addition, we have demonstrated here that these microstructural defects in the wafers can be detected from the thermoreflectance signal, which allows the use of this optical non-destructive method for wafer screening after manufacture.

This work is in part supported by DARPA under Contract No: FA8650-15-C-7517 monitored by Dr. Avram Bar Cohen, supported by Dr. John Blevins, Dr. Joseph Maurer and Dr. Abirami Sivananthan. Any opinions, findings, and conclusions or recommendations expressed in this material are those of the authors and do not necessarily reflect the views of DARPA. DL acknowledges EPSRC for a Research Fellowship (EP/N004493/1) and the Royal Commission for the Exhibition of 1851 for a Brunel Research Fellowship award.

\section{References}

[1] D. C. Dumka, T. M. Chou, F. Faili, D. Francis, and F. Ejeckam, Electron. Lett. 49 (2013) 1298 - 1299.

[2] J.D. Blevins, G.D. Via, K. Chabak, A. Bar-Cohen, J. Maurer, A. Kane, in Proc. CS MANTECH Conf., pp. 105-108, 2014.

[3] D. Francis, J. Wasserbauer, F. Faili, , D. Babić, , F. Ejeckam, W. Hong, P. Specht, and E. Weber., in Proc. CS MANTECH Conf, pp. 133-136, 2007.

[4] D. Altman, M. Tyhach, J. McClymonds, S. Kim, S Graham, J. Cho, K. Goodson, D. Francis, F. Faili, F. Ejeckam, and S. Bernstein, in Proc. ITHERM, pp. 1199-1205, 2014. 
[5] J. W. Pomeroy and M. Kuball, in Proc. CSICS, C.1, 2014.

[6] J. W. Pomeroy, M. Bernardoni, D. C. Dumka, D. M. Fanning, and M. Kuball, Appl. Phys. Lett. 104 (2014) 083513.

[7] D. Francis, F. Faili, D. Babić, F. Ejeckam, A. Nurmikko, and H. Maris, Diamond Rel. Mater. 19 (2010) 229-233.

[8] P.C. Chao, Kanin Chu and Carlton Creamer, in Proc. CS MANTECH Conf., pp. 179-182, 2013.

[9] H. Sun, R.B. Simon, J.W. Pomeroy, D. Francis, F. Faili, D.J. Twitchen, and M. Kuball, Appl. Phys. Lett. 106 (2015) 111906.

[10] J. Anaya, S. Rossi, M. Alomari, E. Kohn, T. Toth, B. Pecz, and M. Kuball, Appl. Phys. Lett. 106 (2015) 223101.

[11] J. Anaya, S. Rossi, M. Alomari, E. Kohn, L. Toth, B. Pecz, K.D. Hobart, T.J. Anderson, T.I. Feygelson, B.B. Pate, and M. Kuball, Acta Materialia 103 (2016) 141-152.

[12]D. Liu, H. Sun, J.W. Pomeroy, D. Francis, F. Firooz, D.J. Twitchen, and M. Kuball, Appl. Phys. Lett. 107 (2015) 251902.

[13] J. W. Pomeroy, R. B. Simon, H. Sun, D. Francis, F. Faili, D. J. Twitchen, and M. Kuball, IEEE Electron Device Lett. 35 (2014) 1007-1009.

[14] S. Karboyan, M.J. Uren, D. Francis, F. Firooz, D.J. Twitchen, and M. Kuball (unpublished). 

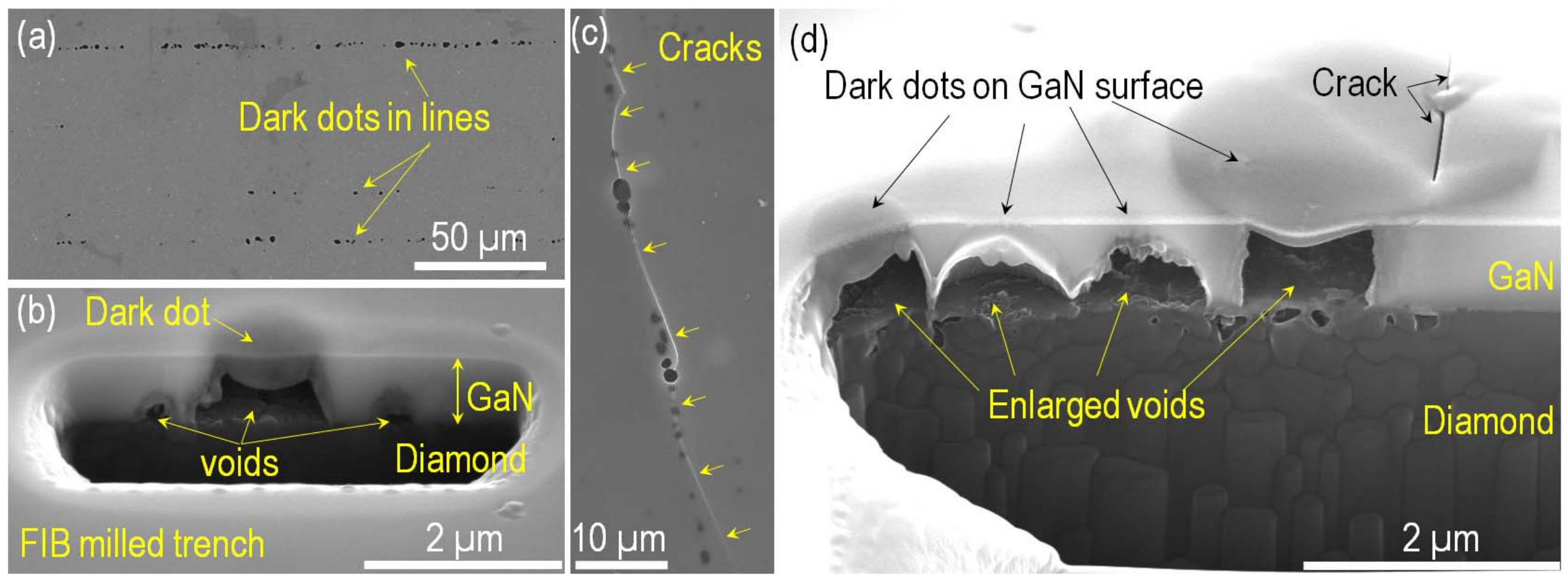

Fig. 1 Scanning electron microscope (SEM) images of as-manufactured GaN-on-diamond grown with 100 nm diamond seeds showing (a) lines of dark dots on the surface of GaN denoted as type A defects; (b) voids at the GaN/diamond interface underneath a typical dark dot in (a); (c) cracks are formed on the surface of GaN along the dark dots after $825^{\circ} \mathrm{C}$ annealing, and (d) the interfacial voids are more pronounced after annealing. 


\section{(a) Top surface of $\mathrm{GaN}$}

\section{$\mathrm{GaN}$}

Dielectric layer

$100 \mathrm{~nm}$
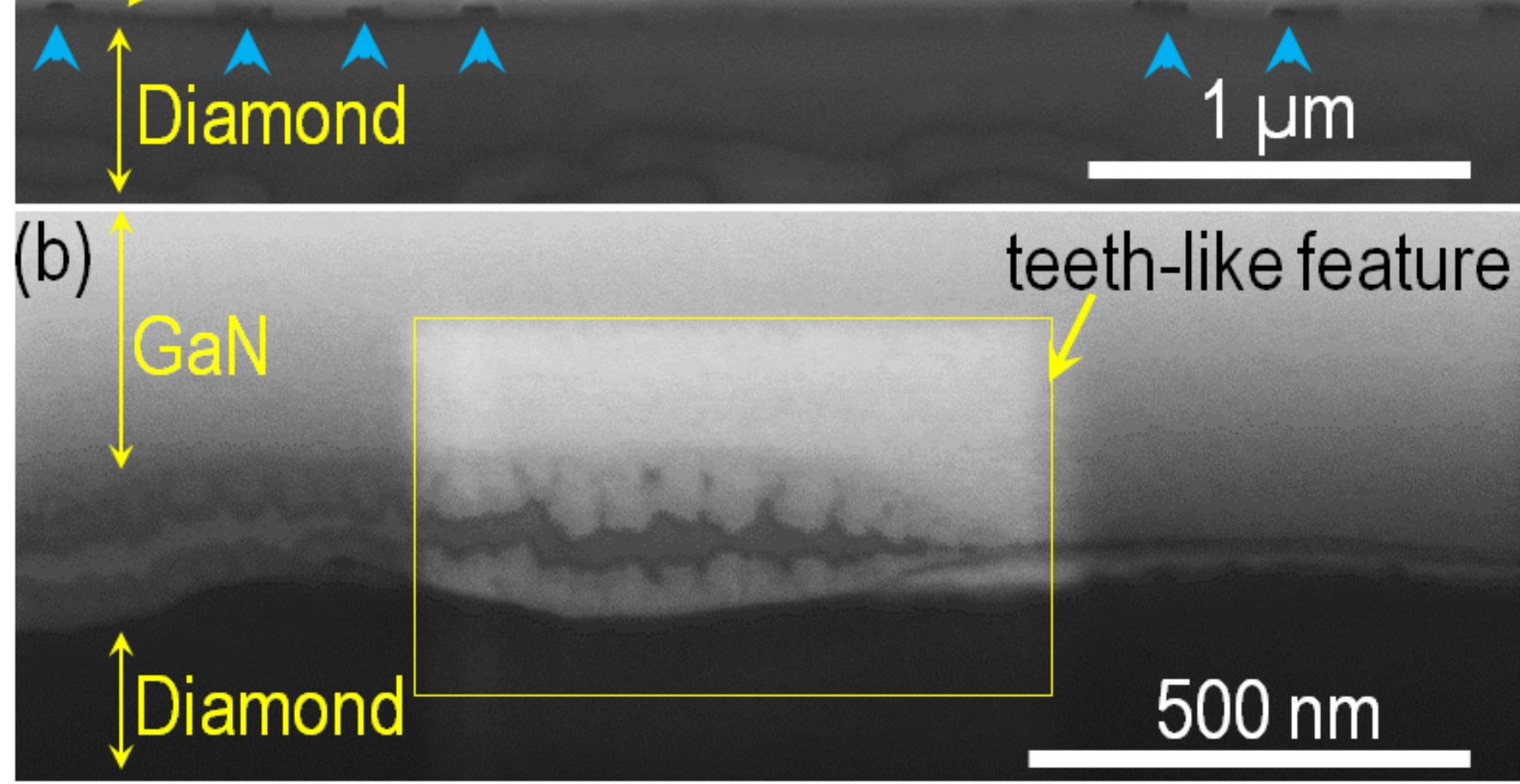

Fig. 2 SEM images showing FIB milled cross-sections of (a) pinholes at the dielectric layer between the $\mathrm{GaN}$ and the diamond in as-manufactured material grown with $100 \mathrm{~nm}$ diamond seeds (type $\mathrm{B}$ defects, marked by arrows; insert is a picture of the pinholes at a larger magnification) and (b) cavity at GaN/ diamond interface with teeth-like features. 
(a) Top surface of $\mathrm{GaN}$

GaN

Dielectric layer

Diamond (b) Top surface of $\mathrm{GaN}$

$1 \mu \mathrm{m}$
GaN

Dielectric layer

$500 \mathrm{~nm}$

$\mathrm{Ga}$

Diamond $500 \mathrm{~nm}$

Fig. 3 SEM images of the cross-section in specimens seeded with $30 \mathrm{~nm}$ diamond particles: (a) as-manufactured and (b) annealed at $825{ }^{\circ} \mathrm{C}$. 

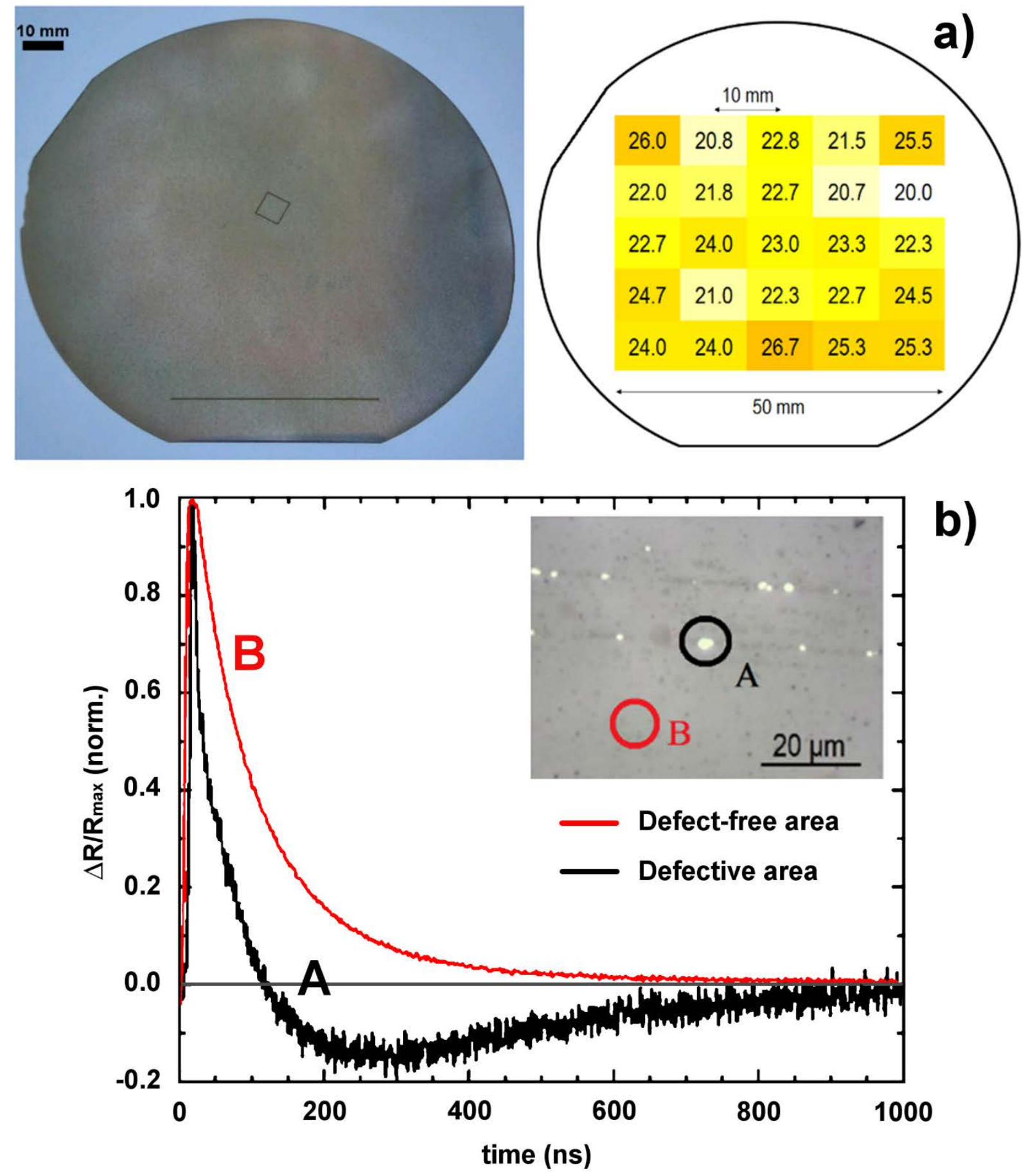

Fig. 4 (a) Image of four inch GaN-on-diamond wafer seeded with 30nm diamond particles and its thermal

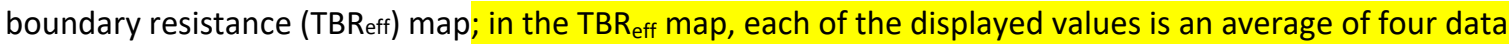
points within a $10 \mathrm{~mm} \times 10 \mathrm{~mm}$ square, with an error bar of $10 \%$; (b) example thermoreflectance transients used to determine TBReff in a defect free area (B) and an area with voids (A) for wafer seeded with $100 \mathrm{~nm}$ size particles, with inset showing optical image of defects and measurement location. 


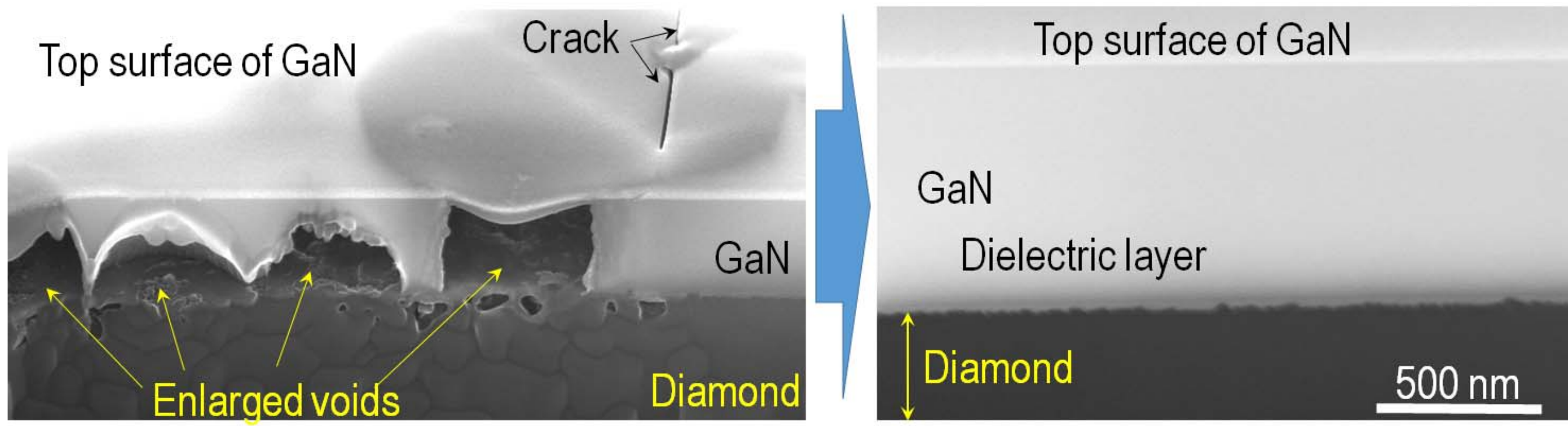

$100 \mathrm{~nm}$ seeded diamond

$30 \mathrm{~nm}$ seeded diamond 